\title{
Permanencia y uso contemporáneo del calendario Cholq'ij/Tachb'al Amaq' en tierras altas de Guatemala
}

\section{Permanence and Contemporary Use of the Maya Calendar Cholq'ij/Tachb'al Amaq' in Guatemalan Highlands}

\author{
Eréndira Juanita Cano Contreras
}

ERin I. J. Estrada Lugo

El Colegio de la Frontera Sur, Sede San Cristóbal, México

Jaime Tomás Page Pliego

Centro de Investigaciones Multidisciplinarias sobre Chiapas y la Frontera Sur, Universidad Nacional Autónoma de México, México

\author{
EgleÉ L. ZeNT
}

Instituto Venezolano de Investigaciones Científicas, Caracas, Venezuela

\begin{abstract}
Resumen: En las tierras altas de Guatemala el conocimiento y uso del Cholq'ij, calendario ritual de 260 días, ha persistido entre grupos mayenses contemporáneos. Si bien no puede afirmarse que sus características han permanecido inalteradas desde la época precolombina, sí se trata de un elemento cultural cuyos principales elementos constitutivos han subsistido a través del tiempo. Sus transformaciones han obedecido a procesos sociales, políticos e históricos que reflejan la capacidad de adaptación de los pueblos mayas de Guatemala, y actualmente el Cholq’ij es el eje de reivindicaciones y propuestas culturales endógenas. En este escrito describimos algunas de sus principales características y elementos constituyentes tal como es comprendido, transmitido, sistematizado y utilizado actualmente entre grupos de las tierras altas mayas de Guatemala. Asimismo, reflexionamos acerca de sus alcances y aplicaciones contemporáneas.
\end{abstract}

Palabras clave: Panmayismo; sistema calendárico; Nawalib’; ajq’ij; cronología maya. 
ABSTRACT: In the highlands of Guatemala, the knowledge and use of the Cholq'ij, 260day ritual calendar, has persisted among contemporary Maya groups. Although it has not remained unchanged since pre-Columbian times, its characteristics do not show significant modifications and its constituent elements have subsisted through time. Its transformations have obeyed social, political and historical processes; this demonstrate the capacity of adaptation of the Maya people of Guatemala. At present the Cholq'ij is the axis of references and endogenous cultural proposals. This paper describes some of its main characteristics and constituent elements. In adition, we explain the way that this calendar is understood, transmitted, systematized and used among the Mayan people from the highlands of Guatemala. Likewise, we discuss on its scope and contemporary applications.

KeYwords: Pan-Mayan movement; calendar system; Nawalib'; ajq'ij; Maya chronology.

ReCEPCIÓn: 15 de mayo de 2019.

ACEPTACIÓN: 26 de agosto de 2019.

Dor: https://doi.org/10.19130/iifl.ecm.2020.56.2.0007

\section{Introducción}

Existe una gran cantidad de escritos, estudios, interpretaciones y análisis sobre los sistemas calendáricos mesoamericanos precolombinos y actuales; probablemente los que versan sobre la zona maya representan el mayor porcentaje. Ante tal diversidad y riqueza de posturas y propuestas es complicado elaborar una síntesis que refleje a cabalidad las concepciones, la medición y la concepción del tiempo, sobre todo por su relevancia en las cosmovisiones locales. En el presente texto deseamos esbozar una síntesis de las principales características del Cholq'ij, partiendo de la descripción general de los sistemas calendáricos mesoamericanos, para posteriormente describir sus características y componentes, además de presentar un panorama actual acerca de usos, concepciones e implicaciones en aspectos sociales, culturales y políticos de pueblos mayas contemporáneos del altiplano guatemalteco.

\section{Sistemas calendáricos mesoamericanos}

En Mesoamérica prehispánica existió un sistema calendárico que, con variantes locales, culturales y linguiísticas, abarcaba toda el área (Tedlock, 1982; Edmonson, 1995; Rupflin, 1999) y fue uno de los elementos etnográficos en que se basó Kirchhoff para definirla como súper-área cultural (Ayala, 2001). Aunque no se ha alcanzado un consenso sobre la fecha aproximada en que se comenzó a usar el sistema calendárico mesoamericano, algunos de los datos más tempranos apuntan a mediados del Preclásico Medio (1200-400 a.C.), pues en este periodo se han fechado sus primeros vestigios, encontrados en las culturas olmeca y zapoteca 
(Edmonson, 1995). Se ha demostrado que algunos calendarios precolombinos al parecer representaban religiones imperiales y, por lo tanto, multiétnicas. En ocasiones, las diferencias en los calendarios reflejaban las rupturas religiosas de grupos étnicos que habían estado unificados. Edmonson (1995) afirma que el calendario k'iche" fue utilizado por representantes de siete lenguas y que los grupos kaqchiqueles usaron al menos dos calendarios.

La creación de sistemas de conteo del tiempo, tanto en lo tocante a los ciclos lunar y solar, como en la llamada Cuenta Larga es una creación mesoamericana. Los registros más tempranos de la Cuenta Larga se atribuyen a la cultura epiolmeca o istmeña, perteneciente probablemente a la familia linguística mixezoqueana ubicada en Veracruz, Tabasco y el suroccidente de Chiapas, aunque también se han encontrado registros en grupos relacionados con la cultura temprana que floreció en lzapa, igualmente de origen mixe-zoqueano (Pérez Suárez, 2012).

La característica fundamental de los sistemas calendáricos comunes al ámbito mesoamericano es que se basaban en un sistema numérico vigesimal. Éstos se componían - y componen - tanto de signos numerales como de glifos calendáricos específicos, cuya combinación hace referencia a un determinado ciclo (Rodríguez, 2006). Los dos ciclos temporales fundamentales eran el denominado "año solar" con duración de 365 días y otro nombrado por algunos "año lunar", de 260 días. Sin embargo, existían ciclos temporales más pequeños de 9, 13, 20 días y ciclos más grandes, de 584, 2920, 18980, 37960, 144,000 o más días, en lo que se ha denominado la Cuenta Larga. El ciclo largo de mayor relevancia es la Rueda Calendárica de 52 años, presente entre todos los grupos mesoamericanos, sobre todo en las metrópolis (Tedlock, 1982; Coe, 1986; Edmonson, 1995; Rupflin, 1999; Rodríguez, 2006).

\section{Sistema calendárico maya}

A la llegada de los españoles, pervivía el uso de sistemas calendáricos en diversos grupos mayenses tanto de tierras bajas como de tierras altas (Coe, 1986; Edmonson, 1995; Weeks, Sachse y Prager, 2009). Se cuenta con registros de algunos de los meses en kaqchikel, así como de una serie de almanaques coloniales: k'iche', kaqchikel y q'eqchi' de Lanquín (Romero, 2000). Igualmente existen referencias españolas sobre el manejo del tiempo entre los lacandones históricos ${ }^{2}$ y algunas

\footnotetext{
${ }^{1}$ En este escrito seguiremos los lineamientos de escritura de vocablos mayas establecidos por la Academia de Lenguas Mayas de Guatemala.

2 Distintos del grupo actual, los lacandones mencionados en las fuentes coloniales conformaban un grupo ch'ol o chortí que habitaba una pequeña isla situada en la laguna Miramar, en el sur de la Selva Lacandona. Sus miembros se autodenominaban "los del Lacam-Tún”, vocablo que, al ser españolizado, se convirtió en lacandón o lacandones (De Vos, 1980; Villa Rojas, 2018). En 1586 el grupo sufrió un intento de reducción por Juan de Morales, siendo la mayor parte aprehendidos o asesina-
} 
descripciones o referencias calendáricas en el Memorial de Sololá, los Anales de los Kaqchikeles, el Chilam Balam de Chumayel y el Chilam Balam de Ixil (Garay y Xoyón, 2016).

La Relación de las cosas de Yucatán de fray Diego de Landa (2003) ${ }^{3}$ es la fuente colonial principal sobre los conocimientos y prácticas mayas en el periodo anterior a la conquista. En este documento se describe el funcionamiento de los periodos calendáricos, registra el nombre de los días y meses de ambas cuentas (365 y 260 días), así como su significado y aspectos simbólicos, además de los conocimientos mayas sobre los ciclos astronómicos de los principales cuerpos celestes, como Venus o Marte. Proporciona información valiosa en el desciframiento y comprensión del sistema calendárico maya y sus datos han sido estudiados e interpretados por importantes mayistas del siglo xx (Thompson, 1927, 1975; Coe, 1986; Freidel, Schele y Parker, 1999).

Otro documento fundamental para el desciframiento del sistema calendárico maya fue la Crónica de Oxkutzcab (Yucatán), que registra una genealogía local en un periodo de tiempo largo. Ésta sirvió como base para la propuesta de correlación de fechas mayas al calendario gregoriano más aceptada en el mundo, desarrollada por Joseph Goodman (1905), revisada por Juan Martínez Hernández (1926), y finalmente corregida por Eric Thompson (1927). A esta correlación actualmente se le denomina GMT 584283, o correlación de Goodman-MartínezThompson (Thompson, 1975; Coe, 1986; Rupflin, 1999; Foster, 2002; Garay y Xoyón, 2016). De acuerdo con ella, los mayas colocaron el comienzo de su calendario en la fecha 4 Ahaw 8 Kumk'u, y la correlación GMT iguala esta fecha con el 13 de agosto de 3114 a.C. En años más recientes, la exactitud de dicha correlación ha sido cuestionada y probada por varios medios, incluyendo la datación por radiocarbono de muestras de dinteles jeroglíficos tallados (Foster, 2002: 281).

Al igual que en otras áreas de Mesoamérica, el calendario de la zona maya se componía de una cuenta corta y una cuenta larga. La primera era el resultado de la concatenación de dos sistemas: el Haab' o Ab' de 365 días y el Cholq’ij de 260 días; el primero determinaba el ciclo agrícola mientras que el segundo era la base de la práctica adivinatoria y ritual.

El Haab' tiene como unidad constitutiva los meses de 20 días, que en total son 18, cada uno con un nombre específico. Estos 18 meses de 20 días suman 360 días, a los que se agrega un período extra de cinco días, llamado wayeb' (denominados también por algunas personas como "días fuera del tiempo"), comple-

dos. Villa Rojas (1967) presume que los pocos que huyeron de la destrucción formaron poblados en lugares menos accesibles y con el tiempo se integraron a otros grupos.

${ }^{3}$ El fraile Landa fue responsable de la mayor destrucción de códices, escritos, imágenes y objetos sagrados en la zona maya, incinerados en una gran pira durante el "auto de fe de Maní", acaecido en ese pueblo de Yucatán el 12 de julio de 1562. Al respecto, Chuchiak (2005: 31) registró: "Los indígenas observaron cómo el religioso ordenaba la destrucción y quema de más de 5000 ídolos y otras parafernalias rituales. A la par de las imágenes se destruyeron más de 27 códices mayas escritos en papel de corteza y piel de venado". 
tando así un año de 365 días. ${ }^{4}$ Sobre el Ab' o Haab' existen referencias sobre su conocimiento y uso entre los pueblos mayas de Guatemala a inicios del siglo xx (Garay y Xoyón, 2016). Hemos registrado que entre grupos k'iche's y kaqchikeles de Guatemala aún hoy en día el año nuevo $\mathrm{Haab}^{5}$ es una celebración importante dentro de la práctica de la espiritualidad maya, sobre todo en espacios organizativos y políticos. En este contexto, los ajq'ijab modernos conceptualizan los wayeb' como un periodo de reflexión, recogimiento e introspección y se refiere que cada uno de los cinco días tiene un propósito específico. ${ }^{6}$

Para Freidel, Schele y Parker (1999) existe una correlación profunda y directa entre la cosmovisión maya del Clásico y Posclásico Temprano con diversos cuerpos celestes, reflejándose en ciclos y características calendáricas. Actualmente se reconoce que no existe tal relación al nivel de profundidad que se creía o que es sumamente difícil demostrarlo (Foster, 2002). No obstante, en el calendario maya se registraron y analizaron los periodos astronómicos de los principales cuerpos celestes que podían observarse a simple vista y estaban dotados de simbolismos culturales y relaciones temporales, como Venus y Júpiter.

La exactitud y multiplicidad de aspectos tomados en cuenta para realizar los cálculos matemáticos y astronómicos que sustentaron el calendario maya sorprendieron a los conquistadores españoles, quienes debieron reconocer su precisión. En las tierras bajas mayas de la península de Yucatán, Diego de Landa registró que quienes habitaban ese territorio habían diseñado un calendario y mencionaba que:

Tienen su año perfecto como el nuestro, de 365 días y seis horas. Divídenlo en dos maneras de meses, los unos de a 30 días, que se llaman U, que quiere decir luna, la cual contaban desde que salía nueva hasta que no parecía.

Otra manera de meses tenían de a 20 días, a los cuales llaman Uinal Hunekeh; de éstos tenía el año entero 18, más los cinco días y seis horas. De estas seis horas se hacía cada cuatro años un día, y así tenían de cuatro en cuatro años el registro de 366 días. Para estos 360 días tienen 20 letras o caracteres con que los nombran, dejando de poner nombre a los otros cinco, porque los tenían por aciagos y malos (Landa, 2003: 138).

Aunque algunos epigrafistas y arqueólogos afirman que no existieron ajustes que solventaran la duración exacta del año solar (poco más de los 365 días),

\footnotetext{
${ }^{4}$ Este periodo de 365 es muy cercano a la cifra equivalente a un año solar, que es de aproximadamente 365.25 días. No se conocen evidencias sólidas para afirmar que existiera algún tipo de corrección anual equivalente al año bisiesto del calendario gregoriano, por lo que el calendario siempre se mantenía con la misma cifra de 365 días (Foster, 2002: 252).

${ }^{5}$ Trabajo de campo, 2015-2018.

${ }^{6}$ Es difícil reconocer el origen de tales creencias y prácticas puesto que, al parecer, esta concepción procedía de las tierras mayas peninsulares y ha sido adoptada durante los procesos contemporáneos de reforzamiento y revitalización cultural, así como de reinterpretación de los sistemas calendáricos.
} 
podemos ver que Landa registra que sí existía este ajuste, aunque es difícil determinar si se trata de una práctica posterior a la llegada de los españoles. En varias páginas, Landa ofrece una descripción sumamente detallada de la composición y funcionamiento del sistema calendárico maya, además de relatar los rituales, creencias, aguieros y festividades relacionadas con los veinte componentes (a los que llamaba "letras" y que equivalen a los 20 Alaxïk o Nawalib' del Cholq'ij) del calendario ritual.

Igualmente, a Landa debemos las primeras descripciones de los ciclos que componen la cuenta larga calendárica, y en su relación explica:

No sólo tenían los indios cuenta del año y de los meses [...] sino que tenían cierto modo de contar los tiempos y las cosas por edades, las cuales hacían de veinte en veinte, contando trece veintes con una de las 20 letras de los meses [¿días?] que llaman Ahau... (Landa, 2003: 139).

Para Edmonson (1995), el sistema calendárico maya puede considerarse único en su tipo, tanto por el grado de sofisticación alcanzado - respecto a los complejos ciclos que lo conformaron- como por la magnitud y precisión de sus cómputos, incluyendo las fechas míticas en "tiempo profundo" que involucran millones de años. El carácter portentoso de este sistema calendárico y su permanencia provoca un sentido de orgullo identitario y de pertenencia entre los mayas guatemaltecos contemporáneos. Fue precisamente en las tierras del altiplano de Guatemala en donde más se conservó el conocimiento calendárico, base de la cosmovisión maya actual, las prácticas rituales y cívicas comunitarias, individuales y familiares (Tedlock, 1982: 2). Se considera que el calendario de 260 días —el más importante y el más usado— ha sufrido pocas modificaciones desde hace varios siglos.

\section{Cholq'ij/Tachb'al Amaq, calendario maya ritual contemporáneo}

El conocimiento y uso del calendario ritual denominado Cholq'ij o Cholb'al Q'ij, en k'iche' y kaqchikel, o Tachb'al Amaq' en ixil ha sobrevivido en gran parte de las comunidades mayas de Guatemala (Tedlock, 1982; Molesky, 2006; Piazza, 2012; Estrada, 2014). La denominación calendárica k’iche' Cholq'ij está compuesta de los vocablos chol- que significa contar cosas puestas en orden y q'ij con el que se designa al día y al sol (Sac Coyoy, 2007: 2). El vocablo cholb'al q'ij, ${ }^{7}$ con el que también se denomina al calendario ritual, se ha traducido como "el ordenador de los días" y se compone de chol, traducido como "orden", b'al que significa "instrumento", y q'ijj, que se traduce como día, tiempo, cumpleaños o misión (García, Curruchiche y Taquirá, 2009).

\footnotetext{
${ }^{7}$ También lo hemos encontrado referido como cholb'äl q'ij.
} 
En ixil al calendario de 260 días se le denomina Tachb'al Amaq' y se compone de los vocablos tachb'al, contar, y amaq', que denomina ciclos, periodos o agrupaciones, pero únicamente en referencia a periodos o grupos de cosas que hay en la naturaleza. De acuerdo con miembros de la Academia de Lenguas Mayas de Guatemala, esta última es una palabra cuyo significado es únicamente comprendido por especialistas rituales y calendáricos. ${ }^{8}$ Aunque existen variantes en los nombres que recibe el calendario en las zonas chuj, q'eqchi' y q'anjobal (La Farge y Byers, 1997; Piedrasanta, 2009), el vocablo más difundido entre mayas de tierras altas es el de origen kiche', Cholq'ij.

En referencias etnográficas de mediados del siglo xx se registró que varios grupos mayas en Guatemala y Chiapas mantenían el uso o al menos algún conocimiento de los calendarios Haab' y Cholq'ij. Estos registros se presentaron en ciertos pueblos de Chiapas (Gossen, 1980), pero sobre todo entre los grupos mayas de Guatemala: popti' (La Farge y Byers, 1997), q'anjob'al (La Farge, 1994), chuj (Piedrasanta, 2009), k'iche (Tedlock, 1982) e ixil (Lincoln, 1942; Colby y Colby, 1981), entre otros. Actualmente, en Chiapas se han perdido los conocimientos sobre calendarios precolombinos, y en Guatemala existe una reivindicación de estos saberes, que hasta hace un par de décadas estaban ocultos o eran resguardados y transmitidos con sumo recelo.

Con algunas adecuaciones y modificaciones, lógicas ante las dinámicas culturales y su transformación en el devenir histórico de los mayas, básicamente se trata del calendario de origen precolombino estructurado en 13 veintenas de días. La rueda calendárica del Cholq'ij se compone del engranaje entre un ciclo de 20 días, representados cada uno por "el rostro de los días" (en sentido simbólico) o "la energía" 9 de cada uno de los 20 Nawales, Alaxik o ahaw, con otro ciclo de 13 numerales. Esta combinación (20 nawales x 13 "niveles de energía" o numerales) es lo que da un ciclo calendárico de 260 días (Tedlock, 1982; Freidel, Schele y Parker, 1999; Gabriel Xiquín, 2000; Cochoy et al., 2006; Sac Coyoy, 2007; Médicos Descalzos, 2012; Craveri, 2013; Estrada, 2014). Así, cada día del calendario se denomina con un nombre compuesto por un numeral de 1 a 13 y uno de los 20 nawalib'/alaxik.

En el uso ritual, adivinatorio y cosmogónico del Cholq'ij actual, los días Nawales, Alaxïk o Nawalib' son el constituyente fundamental. Éstos no son únicamente divisiones cronológicas (Estrada, 2014), como podría asumirse por su acepción de "días": se trata de entidades sagradas con características propias, voluntad, poderes e incluso capacidad volitiva. Aunque existen vocablos específicos en varias lenguas mayenses para nombrarlos, la palabra más frecuente con que se

${ }^{8}$ Pedro Cedillo, Centro Cultural Ixil, Academia de Lenguas Mayas de Guatemala, comunicación personal, 2016.

${ }^{9}$ Aunque esta palabra pueda parecer de corte new age hemos respetado el vocablo en castellano con el cual se refieren a los Alaxïk o Nawales las y los ajq'ijab con quienes trabajamos. Se nos explicó que no se trata de una traducción fiel puesto que estos vocablos no tienen una traducción como tal al idioma castellano. 
denominan actualmente en la oralidad contemporánea de los mayas de Guatemala es el de Nawal o Nawalib' en plural. También se les nombra ahaw ("dueño", "señor", "padre", dios principal, "abuelo"), sobre todo en contextos rituales o al dirigirse a ellos en súplicas, peticiones y consultas, haciendo referencia a su grandeza, atribuciones y poder sobre las vidas humanas. ${ }^{10}$ Otros vocablos como Alaxïk o q'ij Alaxïk son denominaciones locales —el primero en la zona kaqchikel y el segundo más usado en comunidades k'iche'- para nombrarlos en contextos de respeto ${ }^{11}$ y ritual. ${ }^{12}$

La combinación entre las características propias del Nawal/Alaxïk o rostro del día y uno de los 13 numerales correspondientes, le confiere características particulares a una fecha específica y determinarán cuáles son las actividades y plegarias óptimas para ser realizadas, los potenciales peligros y el desarrollo que podrán tener los acontecimientos en el contexto de un evento determinado. Estos días establecen fechas rituales ${ }^{13}$ y actividades propiciatorias para temas específicos, además de que determinan las características, virtudes, potencialidades y "misiones" de la persona que nace en dicho día (Tedlock, 1982; Gabriel Xiquín, 2000; Molesky, 2006; Mendizábal, 2007; Sac Coyoy, 2007; Médicos Descalzos, 2012; Piazza, 2012).

Las características calendáricas de nacimiento de acuerdo al Cholq’ij determinan el potencial de una cierta persona para ser especialista ritual, calendárico o médico, aunque también influyen otros procesos, señales y "llamados"; esto es, la presencia del don o lo que en la zona k'iche' se denomina q'ij aläxik, don o vocación (Médicos Descalzos, 2012), o samaj, por algunos ajq’ijab kaqchikeles.

El conflicto armado interno que padeció Guatemala ${ }^{14}$ tuvo una gran repercusión en diversos aspectos culturales de las comunidades mayas (Arzobispado de Guatemala, 1998) y el uso del Cholq’ij fue condenado e incluso perseguido, así como sus especialistas rituales. Después de la firma del Acuerdo de Paz (1996) se

${ }^{10}$ Armando Zarazúa, comunicación personal, 2016.

11 Según Urdapilleta y Mejía (2015), el uso común del término nawal, nawali' o nawalib' en algunas áreas del territorio maya se ha dado en personas que aún no están inmersas en la práctica de la cosmovisión maya ni en el uso de algún idioma maya. No obstante, en trabajo de campo hemos podido constatar que existen algunas diferencias entre las palabras usadas por distintos/as ajq'ijab. Existen quienes, aunque tengan como lengua materna algún idioma maya, usan comúnmente el término Nawal; mientras que esta palabra es incluso rechazada por aquellos/as que son considerados más tradicionalistas. Por acuerdo y respeto, se utilizan ambos términos: Alaxïk y Nawal, con mayúscula inicial en concordancia con las peticiones de reivindicación cultural.

12 Luis Morales, comunicación personal, 2018.

13 Por ejemplo, el festejo más importante en la espiritualidad maya es el Wajxakib' B'atz' (8 B'atz'), día en que las y los ajq'ijab (sobre todo los hombres) celebran y reafirman su Patan Samaj o sagrada misión. Ese día se entregan las nuevas misiones a ajq'ijab recién iniciados y es un día propicio para festejar matrimonios. Cuarenta días después se celebra el Belejeb B'atz' (9 B'atz') que tiene connotaciones similares, pero enfocadas a la misión de las ajq'ijab mujeres (Gloria Salazar y Armando Zarazúa, comunicación personal, 2016).

${ }^{14}$ De acuerdo con la Comisión para el Esclarecimiento Histórico (1999), el conflicto armado interno abarcó de 1962 a 1996, por lo cual duró 34 años. 
reforzó el proceso de emergencia pública de la cosmovisión y la espiritualidad maya, con el Cholq'ij como una de sus principales manifestaciones (Molesky, 2006; Macleod, 2013). Desde entonces ha existido un repunte en el número de especialistas rituales, calendáricos y médicos mayas, puesto que ya no son perseguidos por el ejército y los grupos paramilitares e incluso se les ha conferido prestigio y estatus en organizaciones mayas. Es de notar que se establece su relevancia y protección en el Acuerdo sobre Identidad y Derechos de los Pueblos Indígenas de Guatemala (1995). Como resultado de reflexiones endógenas, muchos/as ajq'ijab reconocen la importancia de que sus conocimientos sean compartidos y transmitidos, previendo una posible pérdida futura, como la que ocurrió a causa de la violencia. Ello ha resultado en que inclusive se haya dado una apertura para compartir los procesos formativos con personas mestizas y/o extranjeras. ${ }^{15}$

Las y los especialistas calendáricos de mayor edad que sobrevivieron a las masacres continúan enseñando a quienes están en la disposición de aceptar su don y tienen la disciplina necesaria para recibir una gran cantidad de conocimientos transmitidos principalmente de manera oral y adquirir la destreza manual y corporal a través de enseñanzas prácticas. No obstante, la apertura de sus procesos formativos a mestizos y extranjeros, así como la traducción más comúnmente usada de la palabra ajq'ij como "guía espiritual", les ha significado la descalificación de algunos sectores sociales - con mucha frecuencia académicos no mayas y extranjeros-, quienes han calificado ciertos aspectos de su práctica como new age, "invención", "folklorización”, y hasta mercantilización de la cultura.

Más allá de estos conflictos y desencuentros, numerosos ajq'ijab han establecido diálogos e intercambios con estudiosos nacionales y extranjeros, a fin de conocer aspectos históricos, arqueológicos y epigráficos de los sistemas calendáricos mayas precolombinos, contrastándolos con sus propias concepciones y prácticas. ${ }^{16}$ De este ejercicio ha surgido una apropiación de términos e interpretaciones que demuestran el dinamismo y la capacidad de reinterpretar y enriquecer conocimientos occidentales por parte de numerosos especialistas rituales mayas.

No obstante, los estudios arqueológicos y epigráficos no necesariamente han proporcionado una explicación completa acerca del fundamento del calendario de 260 días (Rupflin, 1999). Para un sector de ajq'ijab, "el Cholq'ij dura el tiempo que tarda la Luna en darle nueve vueltas a la Tierra” (Sac Coyoy, 2007: 5), aunque es mucho más frecuente la explicación de que los 260 días que componen el calendario ritual, adivinatorio y sagrado, hacen referencia al tiempo aproximado que dura la gestación humana y también al periodo de crecimiento del maíz - desde su siembra hasta la cosecha- en las zonas altas. En él se refleja la sacralidad de esta planta y su relevancia simbólica en la cosmovisión maya. ${ }^{17}$

\footnotetext{
${ }^{15}$ Gloria Salazar y Armando Zarazúa, comunicación personal, 2017.

16 Armando Zarazúa, comunicación personal, 2017.

17 Trabajo de campo 2015-2018.
} 


\section{Nawalib o Alaxïk, principales constituyentes del Cholq'ij}

Aunque existen detractores que critican el término y uso ritual de los alaxïk o nawales (como son más comúnmente llamados) y los califican de una reinvención moderna, existen datos históricos sobre prácticas y el registro de vocablos muy similares a los que se usan actualmente.

Al respecto, Landa registró que:

Nacidos los niños los bañan luego [...] iban con ellos al sacerdote para que les viese el hado (nawal) y dijese el oficio que debía de tener y pusiese el nombre que debía de llevar el tiempo de su niñez [...] (Landa, 2003: 58).

Sobre este tema también existe una gran cantidad de información disponible, cuya calidad puede variar considerablemente. Debido a que el conocimiento del Nawal o energía de nacimiento de una persona desde el Cholq'ij ha sido -errónea y pobremente- equiparada con una especie de oráculo o hasta astrología maya, es muy común encontrar manuales y páginas en internet que difunden información relacionada. Algunas, no llegan a la media docena, están administradas por grupos, colectivos o confederaciones de ajq'ijab, pero la gran mayoría ofrecen información con frecuencia simplista y esotérica. ${ }^{18}$

Mas allá del uso posmoderno de los Alaxïk, un aspecto fundamental en la espiritualidad y la medicina maya es el conocimiento, consulta y plegaria a los 20 Nawales/Alaxïk del Cholq'ij. Como contadores del tiempo, las y los ajq'ijab conocen con profundidad los procedimientos rituales necesarios para pedir a los 20 ahaw o Alaxik, o al que corresponda con el día o tema en que se está realizando el ritual, que acompañen los discernimientos necesarios para dilucidar el origen, características y tratamiento a seguir en el padecimiento consultado o la situación específica en la que se necesite su ayuda y protección. Igualmente, los ajq’ijab basándose en los Alaxïk determinan los días, lugares y rituales propicios para procesos de atención a la salud, consultas comunitarias, políticas, sociales y otros aspectos de la vida personal, familiar y colectiva.

Se podría inferir que el vocablo Nawal tiene relación con el término "nahual", que hace referencia a la noción mesoamericana de nahualismo ${ }^{19}$ y a su vez está

\footnotetext{
${ }^{18}$ Un ejemplo de ello es la "interpretación" del calendario maya propuesta por José Arguielles en su libro El factor maya (1993). Sin mayor base del conocimiento tradicional del Cholq'ij, propuso un oráculo, que afirmaba estaba basado en el Tzolkin y se componía de 20 kines que combinados con 13 "tonos" establecen una especie de "signo maya". En trabajo de campo hemos podido corroborar el enojo e indignación que ha provocado entre ajq'ijab esta comercialización tergiversada del calendario sagrado maya.

19 Retomando la síntesis conceptual de Martínez (2010: 413) "el vocablo [de origen] náhuatl nahual, nahualli o nagual [hace referencia] a dos nociones diferentes". En un sentido, alude a un especialista ritual con la capacidad de cambiar su forma corporal a voluntad. El otro sentido es el uso de estos vocablos para designar al alter ego, compañero o doble animal que se encuentra íntimamente ligado a una persona desde su nacimiento, compartiendo características de personalidad y un destino común (González, 2001; Martínez, 2010).
} 
muy relacionada con el concepto de "tonalismo". Esta idea podría tener como base un estrecho vínculo que mantuvieron los grupos del altiplano guatemalteco con pueblos nahuas desde el periodo Clásico. Incluso existe una álgida polémica sobre el posible origen náhuatl de grupos como el kaqchikel y el k'iche', idea que ha sido rebatida por etnohistoriadores y epigrafistas (Van Akkeren, 2012). Estos aspectos históricos y el uso de otros vocablos distintos usados en idiomas mayas, como el mencionado alaxïk, wäch q’ij (Urdapilleta y Mejía, 2015) o q’ij alaxïk (Médicos Descalzos, 2012), podrían sustentar la creencia de un origen nahua del término Nawal. ${ }^{20}$

Sin embargo, en el interesante análisis linguiístico morfológico sobre los nombres de los Nawales realizado por Juan Zapil Xivir (2007), él sostiene que la palabra Nawal se relaciona con

el término $\mathrm{Na}$ ' que en castellano quiere decir sentir, conocer, percibir e intuir. [...] Las y los Chuchqajawib' adquieren sus conocimientos a través de la capacidad de $N a^{\prime}-[\ldots]$. El sonido $-w$ marcador de posesión del sustantivo - al, hija o hijo, término usado únicamente por la mujer. De acuerdo a la regla gramatical del idioma K'iche' el juego "A" o ergativo determina todo sustantivo que inicia con vocal, al ser poseído por la primera persona singular se utiliza el sonido " $w$ ". De este análisis morfológico se puede concluir que el término "nawal" quiere decir etimológicamente "hija o hijo, sienta, perciba, conozca, intuya". La palabra $N a$ ' al formar un solo término con la palabra wal, pierde la glotal (') (Zapil, 2007: 44-45).

Así, de acuerdo con este linguiista K'iche', la palabra nawal no se encontraría relacionada con vocablos nahuas, sino por el contrario, se trataría de un vocablo eminentemente maya, con lo que pone por tierra un buen número de críticas sobre su uso.

Al explicar las propiedades simbólicas de los Alaxïk/Nawales, con frecuencia se recurre a metáforas o nociones de autoridad. Barbara Tedlock (1982) refiere que los ajq'ijab k’iche's de Momostenango los denominan "los señores de los días” y se les concibe más que como nombres propios, como nombres divinos. Es por ello que, al referirse a ellos en consultas, ceremonias o discernimientos, se les llama ahaw o ajaw, título de respeto (Tedlock, 1982) o vocablo usado para designar a la deidad superior, Tzakol-B'itol, Creador y Formador, la cual actualmente es concebida como una fuerza o divinidad suprema que contiene la dualidad masculina/femenina en sí. En ocasiones, algunas (y algunos) aqj'ijab que se han acercado a procesos de reflexión y perspectiva de género, mencionan en plegarias e invocaciones: "Gran ahaw, creador-creadora, formadora". ${ }^{21}$ Estos nombres son usados y concebidos de tal forma que no pueden ser traducidos mediante glosas o etimologías. Más bien son interpretados,

\footnotetext{
${ }^{20}$ Por cuestiones de espacio y considerando que desvía la atención del tema principal, además de que merecería un análisis linguístico y etnohistórico a profundidad, en este escrito no ahondaremos al respecto.

${ }^{21}$ Lo cual hemos podido corroborar y registrar en diversas xukulem o "ceremonias mayas".
} 
por medio de frases nemónicas que combinan los significados de los días de acuerdo con las acciones sociales que los caracterizan [...] En la práctica real los nombres se "leen" no como palabras en sí mismas, sino como una especie de glifo oral para otras palabras muy distintas; estas otras palabras se vinculan al nombre del día por medio de la paronomasia, es decir, por medio del juego sónico poético (Tedlock, 1982: 91).

En los procesos de reivindicación y revitalización de diversos aspectos de la cultura maya (como el derecho y la medicina), un aspecto fundamental es la comprensión de las energías que conforman el Cholq'ij. Es por ello que en manuales y propuestas se incluyen algunos de los aspectos principales de los 20 Alaxïk. Frecuentemente estas descripciones no se basan en datos provenientes de la epigrafía, la etnología, la etnohistoria, la antropología o la arqueología, sino de los conocimientos ancestrales e interpretaciones de las y los ajq'ijab que acompañan los diversos procesos. Ello queda manifiesto en el análisis morfológico y linguiístico de los vocablos usados para designar a los 20 Nawales realizado por Zapil (2007). Debido a que brinda una de las descripciones más sintéticas y objetivas sobre el significado de los Nawales, hemos enriquecido las explicaciones que nos han brindado ajq'ijab kaqchikeles, k'iche's e ixiles con sus argumentaciones y propuestas. Al ser conceptos tan complejos, es difícil abarcar sus numerosos significados, simbolismos y acepciones, aunque hemos incluido explicaciones sintetizadas por los aj'ijab. Al analizar ambos acercamientos, resultaron evidentes las convergencias y diferencias entre las diversas formas de comprender los Alaxïk. ${ }^{22}$

En síntesis, los 20 Aläxik o Nawales del calendario ritual Cholq’ij, son (en orden):

1. B'atz: Relacionado con los términos b'aatz, que quiere decir mono saraguato (Alouatta palliata), y ajb'aatz', "quien conoce y ejerce el oficio de la danza de los b'aatz". A este Nawal se le asocia con la palabra b'atz', que quiere decir hilo (Zapil, 2007: 46-47) y debido a que es el primero de la cuenta, es el símbolo relacionado con el hilo del tiempo, que se enrolla y desenrolla. Su enseñanza metafórica es no enredar la propia vida y observar los tejidos. Es el guardián de los artistas y las mujeres que hacen su telar. ${ }^{23}$

2. Ee: Deriva directamente de b'e, “camino" en k'iche'. Por ser un sustantivo, no permite derivaciones, y su significado se asocia con otros vocablos

\footnotetext{
${ }^{22}$ Entre los dos extremos (explicaciones de ajq'ijab que reivindican aspectos "energéticos" de los Alaxïk y acepciones totalmente académicas) se encuentran explicaciones que combinan aspectos académicos con ancestrales, explicaciones de sentido común o conocimiento oral que no se desea que se registre o es únicamente comprendido en la corporalidad. Finalmente, las y los ajq'ijab coinciden en que el conocimiento pleno de los 20 Nawales/Alaxik es una misión casi imposible y a lo largo de su vida, a través de la experiencia y el intercambio con otros/as ajq'ijab, únicamente aumentan su comprensión, aunque nunca será plena.

${ }^{23}$ Síntesis de explicaciones dadas por ajq'ijab kaqchikeles, k’iche's, mames e ixiles; este es el origen de lo expuesto cuando no se especifique otra fuente en las descripciones de los subsecuentes Alaxïk.
} 
relacionados con la acción de conducir, llevar, el concepto de normas y principios orientadores (Zapil, 2007: 49). Hace referencia a caminos cosmogónicos, liderazgos y viajes.

3. Aj: Su marco linguíístico se encuentra en los Aläxik, familias que conforman los patrilinajes y cuya energía se representa a través de la caña del maíz. Hace referencia al jaa, la casa, el hogar. Aaj tiene la interpretación de retomar, procrear, reproducir, multiplicar y reverdecer; es un sustantivo que no permite flexión ni derivación (Zapil, 2007: 51). Se relaciona simbólicamente con la autoridad, la firmeza, el sostén, la vara de autoridad y los niños (como nuevos brotes).

4. Ix: Este vocablo no permite análisis morfológico (Zapil, 2007: 52). Desde un marco conceptual representa el principio femenino y la energía de la Madre Tierra. Simbólicamente se relaciona con el jaguar (Panthera onca), debido a que es un animal cuyo hábitat abarca grandes extensiones territoriales.

5. Tz'ikin: Sobre este Nawal tampoco se encuentran vocablos asociados que permitan el análisis de su morfología linguística (Zapil, 2007: 54). Simbólicamente es el principio de bienestar y la abundancia, la conexión con el ahaw y está profundamente relacionado con las aves, especialmente el águila (diversas especies) y el quetzal (Pharomachrus mocinno). Se relaciona con los sueños, la palabra, la visión y los recursos materiales.

6. Ajmaq: El prefijo aj- es un agentivo, refiere a la persona que tiene un oficio; -maq es oscuridad. También se relaciona con mak, que significa "falta, error, vergüienza" (Zapil, 2007: 58). Se relaciona con lo que está oculto, el conocimiento velado, las faltas, y es el Alaxïk que representa a los antepasados y las bendiciones y consejos que otorgan desde otro nivel de la existencia.

7. N'oj: Se enmarca conceptualmente en el cerebro, con el término n'aoj. $N a^{\prime}$ es un verbo intransitivo que significa "sentir conocer, percibir, intuir" y probablemente es la raíz de la palabra nawal. El grafema -oj es un sufijo de sustantivo verbal (Zapil, 2007: 60). Por tanto, representa la sabiduría que deriva de los sentidos, los conocimientos, las ideas, los pensamientos, las intuiciones y la percepción. Simbólicamente se relaciona con el sagrado conocimiento, no sólo a un nivel mental, sino el que es experiencia, reflexión y práctica, sabiduría. Muestra cómo equilibrar sentimientos y pensamientos para llegar al bienestar físico, espiritual y emocional.

8. Tijax: Se enmarca simbólicamente en la piedra de obsidiana. Se asocia al término k'ax, que quiere decir dolor físico y/o psíquico (Zapil, 2007: 61). Se relaciona con la curación y la medicina, pero también con los sufrimientos y el desequilibrio. Se describe como un cuchillo de doble filo (artefacto de obsidiana) y como tal es usado simbólicamente en contextos rituales.

9. Kawoq: Se compone de los elementos $k$ - tiempo/aspecto incompletivo, $-a$ 
ergativo de tercera persona singular, -woq verbo intransitivo que significa hacer ruido al hablar. Se ha traducido como "trueno". Se puede asociar al término k'aqob'al, con el que se designan algunas enfermedades causadas por algún acto incorrecto de los antepasados (Zapil, 2007: 62-63). Simbólicamente se relaciona con la colectividad, la palabra fuerte y las tortugas (varias especies).

10. Ajpú: Este concepto remite al personaje del Popol Wuj, Jun Ajpu, gemelo de Ixb'alamke', quienes vencieron a los señores de Xibalbá y fueron los primeros abuelos. ${ }^{24}$ También se interpreta desde su constitución por el prefijo $a j$ - que es un agentizador de oficio y -pu o -pub verbo transitivo para cazar, tirar (Zapil, 2007: 65). Debido al relato, que describe al personaje como experto cerbatanero, se relaciona con este instrumento y, por tanto, simbólicamente remite a la precisión y el tino, además de la claridad, la vida y la fuerza.

11. Imox: Se relaciona con los términos imux, cosa oculta, y mox, "no piensa". Conceptualmente representa el agua y se asocia al término moxireem, que significa "molestia, enojo, inestabilidad en pensar" (Zapil, 2007: 66). Simbólicamente se relaciona con las emociones, la locura y los aspectos, y animales acuáticos (peces, cocodrilos), además de la fertilidad y la vida.

12. Iq': Desde el Preclásico se relaciona con las nociones de viento, espíritu, hálito (Freidel, Schele y Parker, 1999). También se asocia a jiq', nombre dado a las pequeñas figuras de piedra. En su derivación se encuentra iq'ilal, que indica un estado de enojo de la persona (Zapil, 2007: 68). Actualmente se le relaciona con el colibrí (diversas especies) y aspectos espirituales y emocionales de la persona.

13. Aq'ab'al: $a q$ 'ab es "noche, madrugada" (Zapil, 2007: 69). Su marco simbólico es la claridad, el amanecer, la aurora y la dualidad presente en los momentos del día en que la oscuridad y la sombra conviven. También se afirma que deriva de las palabras $a q$ 'ab, "oscuridad" o "noche", y b'al, "manto" o "espacio de claridad" (Morales, 2016: 58). Se le relaciona simbólicamente con la casa, como un manto que protege a la familia; además de ser la representación del principio cosmogónico de la dualidad que existe en todos los elementos que integran el cosmos y el equilibrio que debe existir entre ambos aspectos constitutivos.

14. K'at: Es la raíz del verbo "encender" y "ofrendar", k'asaj se le llama a una deuda pendiente (Zapil, 2007: 70). Simbólicamente se relaciona con la red donde se transporta el maíz, que remite a la telaraña y a las cárceles (metafóricas y materiales), además de representar la unión colectiva (figura simbólica de la red y las mazorcas contenidas en ella) y el sagrado fuego.

15. K'an: Remite a diversos tipos de ofidios, entre los que destacan la serpiente de cascabel (Crotalus simus) y la nauyaca (Bothrops asper), probablemente

${ }^{24}$ Ajq’ijab mames y kaqchikeles. 
las víboras de mayor carga simbólica en tierras mayas. Se relaciona con la justicia, el tiempo, los cambios. ${ }^{25}$ También se ha asociado con la palabra k'aan, enojado y k'anaal, enojo (Zapil, 2007: 73).

16. Keme': También nombrado kame. Atañe a la muerte y conceptualmente tiene correspondencia con los señores del Xibalbá mencionados en el Popol Wuj, Jun Keme y Wuqub Keme. ${ }^{26} \mathrm{Kam}$ es la raíz de la palabra "muerte". Se relaciona con kameel, que proviene de kam, raíz, eel, sustantivo agentivo: que ejerce acción de muerte (Zapil, 2007: 73-74).

17. Kiej: En ocasiones se utiliza también el vocablo keej, concerniente a kejaj, que significa montar, cabalgar (Zapil, 2007: 81). Su relación simbólica se encuentra en las cuatro esquinas de los rumbos cósmicos y los cuatro colores del maíz, además del equilibrio cuatripartita del cosmos. Las y los ajq'ijab contemporáneos lo traducen como "venado" y se le considera el guardián de la montaña, el bosque y las selvas.

18. Q'anil: Es un nombre propio con raíz q'an, que significa "amarillo o maduro", con -il, sustantivizador, que da la calidad, "el que hace que madure", es la energía que permite madurar a los frutos. Tiene dos acepciones, una relacionada con la abundancia de vida y otra a la falta de energía y salud (Zapil, 2007: 82-83). Conceptualmente se relaciona con la idea de semilla, simiente, la promesa de los inicios y la nueva vida. En contextos ceremoniales es a quien se encomiendan las semillas de cultivos autóctonos, los embarazos y la simiente humana.

19. Toj: El verbo intransitivo tooj significa reconocer, trascender los dones recibidos. Se relaciona con la actitud de agradecimiento y se representa con la resina de pino en forma de moneda (denominada cuilco). Tooj también significa "súplica, armonización, pago, reconocimiento" (Zapil, 2007: 85-86). Simbólicamente remite al "mandato primordial" (Morales, 2016: 61) de hacer ofrendas de agradecimiento al Creador y Formador y a las entidades sagradas. Es el sagrado fuego (xukulem o ceremonia), la ofrenda de copal, inciensos, candelas, flores. ${ }^{27}$

20. $T z^{\prime} i$ ': $t z^{\prime} i j$ se asocia con la búsqueda de la verdad. El concepto de $t z^{\prime} i{ }^{\prime}$ se asocia con la energía del deseo intenso, katz'i'owik o katz'i'arik; también con el deseo sexual intenso, tz'ilonik (Zapil, 2007: 87-89); relacionado simbólicamente con el coyote, el perro y el tepezcuintle. En contextos rituales se asocia con las peticiones relativas a asuntos jurídicos y procesuales.

La interpretación del significado e implicaciones cosmogónicas de cada uno de los Nawales/Alaxik es variable y depende del área cultural, el contexto en que se esté usando e incluso del o la ajq'ijab, aunque nunca se modifican de manera

\footnotetext{
25 Trabajo de campo 2015-2018.

${ }^{26}$ Ajq'ijab kaqchikeles y k'iche's.

27 Francisca Salazar, comunicación personal, 2015.
} 
profunda pues, en general, hacen referencia a los mismos elementos. Su manejo y comprensión es uno de los aspectos fundamentales en el proceso de formación de los especialistas rituales calendáricos, quienes adquieren estos conocimientos a través de la transmisión oral por parte de quienes les enseñan, a través de sueños o por medio de otras maneras de percepción: estados meditativos, mensajes de maestros o entidades no encarnadas, ensoñaciones, presentimientos y sensaciones corporales.

\section{Ajq'ijab, especialistas rituales calendáricos y su papel actual}

A las y los especialistas rituales calendáricos, esto es, a quienes tienen como sagrada misión el conocimiento, uso y manejo del calendario sagrado Cholq’ij, se les denomina con la palabra k'iche' ajq'ij, compuesta del prefijo aj- que denota oficio o facultad de hacer algo, y q'ij, que, como se dijo, significa "día", "sol", "tiempo" (Sac Coyoy, 2007: 3). Frecuentemente ajq'ij se traduce como "quien cuida/guarda los días, el tiempo" o "el/la contador/a de los días"; en la literatura inglesa se ha traducido como day-keeper (guardián de los días). En la zona ixil a los especialistas calendáricos se les denomina aaq'ii y de acuerdo con investigadores del Centro Cultural Ixil de la Academia de Lenguas Mayas de Guatemala, este vocablo se compone de $a a$, "autoridad, vara", y q'ii, "día, sol"; se traduce como "quien lleva los días". ${ }^{28}$

Las personas que fungen como ajq'ij tienen una gran trascendencia en la transmisión, conservación y práctica de la espiritualidad y la cosmovisión maya. Como se comentó, en términos muy generales, su quehacer se basa en el cálculo de la cuenta del Cholq'ij y la comprensión de los 20 Alaxïk que conforman este sistema calendárico de 260 días. Para poder llevar a cabo su labor, deben conocer profundamente la influencia de cada uno de los Alaxik y su combinación con los 13 numerales en procesos - de salud, emocionales, organizativos, espirituales, políticos, sociales, económicos, relacionales-colectivos e individuales.

Los y las ajq'ijab pueden indagar en los "dones" o características especiales con los que nacen quienes tienen potencial para desarrollar actividades rituales o curativas. En múltiples ocasiones, tal como sucede en una gran cantidad de sistemas etnomédicos, la persona que no desarrolla sus dones, los comparte y/o potencializa para el bien común padece una serie de aflicciones que generalmente le llevan a aceptar su misión (Tedlock, 1982; Médicos Descalzos, 2012) y comenzar su aprendizaje con otro/a especialista, a través de sueños o de diversas vías. ${ }^{29}$

\footnotetext{
28 Pedro Cedillo, Academia de Lenguas Mayas de Guatemala, Centro Cultural Ixil, comunicación personal, 2016.

${ }^{29}$ Como estados meditativos, experimentación con elementos terapéuticos, práctica y experiencia corporal, entre muchas otras, tal como nos han compartido diversos ajq'ijab kaqchikeles, k'iche's, mames e ixiles.
} 
Los ajq'ijab son las personas reconocidas como especialistas en comprender, conocer y contactar las fuerzas espirituales para acompañar y resolver tanto procesos de salud física, mental, energética o espiritual, como situaciones familiares, colectivas, políticas o de cualquier otra índole que tenga la persona, colectivo, familia o pareja que le consulta y puedan estar causando desequilibrios, padecimientos y sufrimientos; ${ }^{30}$ cumplen funciones sociales, culturales y simbólicas de suma importancia (Tedlock, 1982; Gabriel, 2000; Molesky, 2006; Juárez y Puac, 2008); acompañan procesos organizativos, médicos, sociales, culturales y políticos a través de la realización de ceremonias y otros procesos rituales. De acuerdo con la misión que traen asignada desde su nacimiento, hay diversos tipos y especializaciones de ajq'ij. En los espacios de socialización intercultural, se les nombra "guías espirituales mayas" como una forma de distinguir su práctica de otros procesos religiosos y enfatizar su quehacer como parte de la salud espiritual colectiva; pueden ser hombres o mujeres y prácticamente no existe una edad límite para iniciar su camino de aprendizaje; incluso se nos ha referido que, en comunidades tradicionalistas y alejadas, niños en edad escolar y preescolar comienzan a ser formados y reciben su vara'/patan samaj a edades muy tempranas.

El proceso de aprendizaje o "formación" — como comúnmente se denomina en español- de un ajq'ij está marcado por la realización de discernimientos rituales, que generalmente son precedidos por circunstancias adversas en su vida, símbolo de la misión o don que poseen. Este aspecto se encuentra generalizado en el ámbito etnomédico y ritual mesoamericano y marca substancialmente a quienes son ajq'ijab o médicos mayas por nacimiento, de quienes han estudiado por capacitación de organizaciones o en contextos académicos (Consejo Mayor de Médicos Maya'ob por Nacimiento, 2016). ${ }^{31}$

Cuando se ha descubierto la q'ij Alaxïk (o misión sagrada) como fuente de los diversos padecimientos y sufrimientos que tiene una persona, el o la ajq'ij consultado o algún otro comienza el proceso de sanación de la persona enferma. Generalmente inicia con la aceptación del q'ij Alaxik, que marca la necesidad de ayudar a otras personas a través del conocimiento calendárico y la práctica médica en la curación de enfermedades específicas, dependiendo de la especialidad médica con que haya nacido (Médicos Descalzos, 2012: 5). Para conocer cómo desarrollar sus dones y bajo qué especialidades médicas debe desarrollarlos, el o la futura $a j q ' i j$ buscará el consejo de los especialistas que existan en su contexto cultural específico o que estén en posibilidad de enseñarle.

Frecuentemente, el descubrimiento de la vocación por el discernimiento a través de los Alaxïk de nacimiento se encuentra acompañado de otro tipo de señales y eventos sobrenaturales. Siempre existen sueños recurrentes en los que la persona con "don" recibe visitas, paquetes, enseñanzas, consejos y/o palabras de personas con apariencia antigua o que les explican con claridad cuál es su misión

\footnotetext{
${ }^{30}$ Luiz Choy, Gloria Salazar, Armando Zarazúa y J. Chiriz, comunicación personal, 2017.

31 Gloria Salazar, comunicación personal, 2017.
} 
y cómo deben llevarla a cabo. Además, según han sistematizado los Médicos Descalzos de Chinique (2012), en el Quiché existen síntomas del q'ij Alaxïk reflejados en señales corporales y manifestaciones físicas o somáticas, sensoperceptivas ("alucinaciones" visuales y auditivas por escuchar y ver a "los abuelos"), ${ }^{32} \mathrm{del}$ comportamiento, emocionales o trastornos de sueño.

El quehacer de los/as actuales ajq'ijab se ha conformado de manera distinta a las especialidades rituales y etnomédicas que existían tradicionalmente (Piazza, 2012). Se han reportado diferencias entre las prácticas de "las y los nuevos ajq'ijab" con las de curanderos, chamanes o rezadores tradicionales, y hay quien afirma que estos últimos conservan un carácter mucho más íntimo y sobre todo, con reminiscencias de sincretismo católico (Piazza, 2012). En contraste, la nueva vertiente de $a j q$ 'ijab, sobre todo quienes se encuentran más sumergidos en procesos de reivindicación, rechazan la presencia de elementos católicos en sus ceremonias y rituales, argumentado que reflejan el colonialismo religioso al que se ha sometido a la cultura y tradición maya. ${ }^{33}$

Hemos observado el quehacer ritual e intercambiado ideas e información con ajq'ijab tz'utujiles, k'iche's, kaqchikeles, ixiles, mames y q'eqchi'es en el norte, occidente y altiplano central de Guatemala. Estas pláticas y espacios nos han enseñado que esta "nueva" vertiente de ajq'ijab no es un conglomerado homogéneo que comparta las mismas características. Por el contrario, se trata de una colectividad heterogénea, que si bien se agrupa —un tanto artificialmente— bajo la misma denominación en función de su especialidad calendárica, se constituye de especialistas médicos y rituales con características diversas, resultado de su adscripción etnolinguística, historia familiar y colectiva, especialidad médica de nacimiento, participación en procesos políticos, de revitalización o reivindicaciones culturales, procesos de aprendizaje, profesión u ocupación e interacciones con otros especialistas locales.

Así nos encontramos con una profesión marcada por múltiples implicaciones, interpretaciones y dimensiones simbólicas. Aunque algunos detractores consideran que la figura del ajq'ij tiene mucho de reinvención, el trabajo de las y los actuales ajq'ijab puede dilucidarse mediante estudios arqueológicos, etnohistóricos y etnográficos (Molesky, 2006). Se ha relacionado este vocablo con los especialistas rituales llamados aj-k'in, encontrados en registros en la región Puuc; los actuales ajq'ijab pueden ser encontrados en referencias coloniales como los "guardianes de los naguales". Aunque el vocablo en sí no se ha encontrado en registros arqueológicos, se sabe que el oficio de especialistas calendáricos está registrado desde el Preclásico (Piazza, 2012).

\footnotetext{
32 Se denomina así, "abuelos", a diversas entidades sobrenaturales, como deidades de la tierra, espíritus guardianes de sitios sagrados, ancestros, héroes míticos y personas sabias ya fallecidas.

33 María Teresa Mosquera, comunicación personal, 2015.
} 


\section{Permanencia e implicaciones del uso y manejo contemporáneo del Cholq’ij}

El conocimiento, uso y manejo del Cholq'ij y los aspectos calendáricos adivinatorios fueron resguardados durante siglos en las tierras altas de Guatemala, probablemente debido a su remota ubicación geográfica y a las elevadas altitudes en que se encuentran muchas comunidades, lo cual las hizo relativamente inaccesibles para los españoles coloniales, las reformas liberales de la década de 1870 y las visitas pastorales de sacerdotes católicos (Molesky, 2006). Esta permanencia contrasta con la erosión del uso calendárico y la incorporación de elementos católicos a la ritualidad que aconteció en las tierras bajas del este, las cuales tuvieron una intensa actividad española que derivó en procesos de sincretismo. Además, debido a la persecución religiosa y a los procesos sociopolíticos de las décadas recientes, las prácticas rituales y adivinatorias se desenvolvieron en el ámbito privado y se realizaban de manera oculta.

Probablemente el proceso más notable de la ritualidad maya de las tierras altas de Guatemala es la explosión de su visibilidad y la emergencia de prácticas públicas. Como producto del Acuerdo de Paz de 1996, surgió un orgullo de pertenencia y una nueva agencialidad del pueblo maya, que tiene uno de sus aspectos más visibles en la práctica de la denominada espiritualidad maya. En la actualidad, algunos ajq'ijab afirman que probablemente del 40 al 50\% de la población indígena en Guatemala practica alguna forma tradicional ritual, aunque sólo el $10 \%$ lo hace abiertamente. En algunas de las comunidades de las tierras altas mayas más tradicionalistas, se considera que del 45 al $80 \%$ de la gente realiza prácticas tradicionales, mientras que en otras comunidades sólo del 5 al $10 \%$ ha conservado prácticas mayas (Molesky, 2006).

En el trabajo de campo hemos observado diferencias cualitativas en la permanencia de las prácticas rituales por zonas. En Quiché, uno de los departamentos con orografía más accidentada, es reconocida la permanencia y práctica de la ritualidad maya, sobre todo en las zonas k'iche's cercanas a Santa Cruz, Chichicastenango y el área ixil. Lo mismo acontece con el departamento de Sololá y la zona circundante al Lago de Atitlán, aunque en esta última también prosperan movimientos y organizaciones que utilizan aspectos de la cosmovisión y la espiritualidad maya con fines mercantilistas, debido al gran flujo turístico. En las zonas bajas, los departamentos de Cobán, Alta y Baja Verapaz son reconocidos por la importancia de la práctica ritual de numerosas comunidades q'eqchi'es. Por su parte, en las zonas k'iche' y kaqchikel cercanas a núcleos urbanos, como los departamentos de Quetzaltenango, Chimaltenango y Sacatepéquez, puede notarse una gran presencia de iglesias cristianas, a las que pertenece un porcentaje significativo de la población.

Se debe aclarar que, aunque existe un importante movimiento reivindicatorio de las prácticas culturales y rituales mayas, su respeto y reconocimiento por parte de la sociedad nacional es limitado, en parte debido al profundo racismo 
y discriminación que persiste en la sociedad guatemalteca hacia las personas indígenas (Álvarez, 2013). Por otra parte, algunas voces califican esta nueva visibilidad de las prácticas rituales como "cultos neotradicionales" y les confieren un sentido protagónico y en ocasiones incluso mercantilista (Pédron, 2008); otras cuestionan su pretendida diacronía, algunos más las tildan de reinvenciones que parten de la interpretación de textos y estudios arqueológicos y epigráficos. Por el contrario, académicos/as mayas analizan sus implicaciones y potencialidades en términos de justicia epistémica y descolonización del pensamiento. ${ }^{34}$

En tanto actores sociales, la percepción colectiva de las y los ajq'ijab varía en función de diversos factores sociales y políticos. La composición religiosa de la comunidad o el medio en el que se desenvuelven determina el prestigio que pueden - $\mathrm{O}$ no- tener. Hemos observado que en pueblos y ciudades pequeñas de Chimaltenango y Sacatepéquez, donde la presencia de religiones evangélicas es alta, ${ }^{35}$ con frecuencia los y las ajq'ijab son nombrados despectivamente como "brujos" y "brujas", y despiertan sentimientos encontrados: algunas personas les temen; otras les respetan y tienen cariño; algunas más les desdeñan, y la mayoría acude a ellos únicamente cuando han agotado otras vías para solucionar problemas de salud. Por el contrario, en contextos de activismo y reivindicaciones mayas o relacionados con el movimiento maya, se les tiene en alta estima y gozan de un prestigio y respeto prácticamente generalizado. En otros lugares del país, como la zona ixil, su práctica es menos pública y la mayoría guarda cierto anonimato, que la gente respeta. En estos lugares más tradicionalistas es muy raro que alguna persona - aunque pertenezca a alguna religión cristiana — se refiera a ellos con desdén, sorna o disgusto. Por el contrario, se rodean de un halo de callado y discreto respeto.

\section{Consideraciones finales}

El proceso de reflexión, diálogo e intercambio de saberes entre personas mayas y académicos/as extranjeros y nacionales ha conformado espacios de re-apropiación de conocimientos ancestrales, que algunos linguiistas, profesores, ajq'ijab y activistas mayas han calificado como justicia epistémica. Estos hombres y mujeres mayas interesados en su propia historia, han acompañado, generado o participado en procesos de aprendizaje, enseñanza e interpretación de aspectos asociados a la cultura maya, como epigrafía y escritura, matemáticas, arqueología y astronomía.

\footnotetext{
34 Juana López Batzin, entrevista personal, 3 de abril de 2016.

${ }^{35}$ En los años del conflicto armado interno, un aspecto de la táctica del ejército de "quitarle el agua al pez" fue fomentar la entrada de iglesias evangélicas y pentecostales que promovían el individualismo y cortaban con el pasado ancestral "pagano" que había provocado la aparición de grupos armados y por tanto sus desgracias. Estas iglesias además ofrecían oportunidades de rápido y fácil ascenso social y político mediante el ejercicio del ministerio religioso, a la par que comenzaron a apoyar financieramente proyectos de creación de escuelas y hospitales.
} 
Dicho proceso resulta enriquecedor en términos del propio quehacer y las reflexiones que surgen entre ajq'ijab y activistas. Los nuevos conocimientos les permiten conformar explicaciones más complejas sobre creencias e interpretaciones, aspectos rituales, señales y fenómenos meteorológicos. Se conforma así un ejercicio en el que se refuerza el orgullo de pertenencia y se reinterpretan los conocimientos y estudios científicos a la luz del conocimiento y las prácticas tradicionales.

Si bien la permanencia del Cholq'ij en las tierras altas de Guatemala implica la transmisión y cuidado de conocimientos ancestrales transmitidos generalmente de manera oral, como todo producto cultural no se encuentra exento de reinterpretaciones, adecuaciones y cambios. La continuidad del Cholq'ij está permeada por la negociación y apertura de sus custodios a nuevos procesos de aprendizaje y enseñanza que pueden incluir el diálogo con otras cosmovisiones, en aras de enriquecer la propia experiencia y asegurar su permanencia.

\section{Bibliografía}

Acuerdo de Paz firme y duradera

1996 Gobierno de la República de Guatemala. Unidad Revolucionaria Nacional Guatemalteca. Guatemala: Naciones Unidas.

Acuerdo sobre Identidad y Derechos de los Pueblos Indígenas de Guatemala

1995 Gobierno de la República de Guatemala. Unidad Revolucionaria Nacional Guatemalteca. Comisión Política Diplomática (firmantes). México: Naciones Unidas.

Álvarez Díaz, Andrea

2013 "Violencia de género y otras violencias: relatos de mujeres mayas de Guatemala", Nuevas Tendencias en Antropología, 4: 68-103.

Argüelles, José

1993 El factor maya, un camino más allá de la tecnología. México: Círculo Cuadrado.

Arzobispado de Guatemala

1998 Guatemala nunca más. Informe Proyecto Interdiocesano de Recuperación de la Memoria Histórica. Tomo I. Impactos de la Violencia. Guatemala: Oficina de Derechos Humanos del Arzobispado de Guatemala.

Ayala Falcón, Marisela

2001 "La escritura, el calendario y la numeración”, Historia antigua de México. Volumen IV. Aspectos fundamentales de la tradición cultural mesoamericana, pp. 145187, Linda Manzanilla y Leonardo López Luján (coords.). México: Instituto Nacional de Antropología e Historia, Universidad Nacional Autónoma de México, Editorial Porrúa. 
Chuchiak, John F.

2005 "El regreso de los autos de fe: fray Diego de Landa y la extirpación de idolatrías en Yucatán, 1573-1579”, Península, 1: 29-47.

Cochoy Alva, María Faviana, Pedro Yac Noj, Isabel Yaxón, Santiago Tzapinel, Rosenda Camey, Daniel Domingo López, José Yac Noj y Carlos Tamup Canil

2006 Raxalaj mayab' k'aslemalil. Cosmovisión Maya, plenitud de la vida. Guatemala: Programa de las Naciones Unidas para el Desarrollo.

Coe, Michael

1986 Los mayas: incógnitas y realidades. México: Diana.

Colby, Benjamin y Lore M. Colby

1981 The Daykeeper, the Life and Discurse of an Ixil Diviner. Cambridge: Harvard University Press.

Consejo Mayor de Médicos Maya'ob por Nacimiento

2016 Raxnaq'il Nuk'aslemal: Medicina Maya' en Guatemala. Guatemala: Asociación Ati't Ala' y Cholsamaj.

Craveri, Michela

2013 "El calendario adivinatorio de 260 días en la sociedad maya contemporánea: usos, funciones y estructura", Otras Modernidades, 7: 14-36. Dol: https://doi. org/10.13130/2035-7680/3065.

De Vos, Jan

1980 La paz de Dios y del Rey: la conquista de la Selva Lacandona (1525-1821). México: Secretaría de Educación y Cultura de Chiapas, Fondo de Cultura Económica.

Edmonson, Munro

1995 Sistemas calendáricos mesoamericanos. El libro del año solar. México: Universidad Nacional Autónoma de México.

Estrada Peña, Canek

2014 "Anima' ri cho, Anima' ri plo: espíritu de la laguna, espíritu del mar. Acerca del día Imox entre los k'iche'”, Estudios de Cultura Maya, XLV: 191-224. DoI: 10.19130/iifl.ecm.2015.45.138.

Foster, Lynn

2002 Handbook to Life in the Ancient Maya World. Nueva York: Facts on File.

Freidel, David, Linda Schele y Joe Parker

1999 El cosmos maya, tres mil años por la senda de los chamanes. México: Fondo de Cultura Económica.

Gabriel Xiquín, Calixta

2000 Función social de guías espirituales maya kaqchikeles, tesis de licenciatura en Trabajo Social. Guatemala: Universidad Rafael Landívar. 
Garay, Alejandro e Igor Xoyón

2016 Armonización de los calendarios mayas. Guatemala: Ministerio de Cultura y Deportes.

García, Pablo, Germán Curruchiche Otzoy y Simeón Taquirá

2009 Ruxe'el Mayab' K'aslemäl, Raíz y espíritu del conocimiento maya. Guatemala: Programa de Educación Intercultural Bilingüie de Centroamérica, Universidad Rafael Landívar y Consejo Nacional de Estudios Mayas.

González Torres, Yólotl

2001 Animales y plantas en la cosmovisión mesoamericana. México: Plaza y Valdés, Instituto Nacional de Antropología e Historia y Sociedad Mexicana para el Estudio de las Religiones.

Goodman, Joseph T.

1905 "Maya Dates", American Anthropologist, 7 (4): 642-647.

Gossen, Gary

1980 Los chamulas en el mundo del sol. Tiempo y espacio en una tradición oral maya. México: Consejo Nacional para la Cultura y las Artes, Instituto Nacional Indigenista.

Juárez Pú, Sebastiana C. y Nicolasa Puac Pech

2008 Espiritualidad maya en Totonicapán (casos específicos de las aldeas Chuatroj, Vásquez, Chipuac y Pasajoc). Guatemala: Proyecto Linguiístico Santa María.

La Farge, Oliver

1994 La Costumbre en Santa Eulalia, Huehuetenango en 1932. Guatemala: Cholsamaj, Ediciones Yax Te'.

La Farge, Oliver y Douglas Byers

1997 El pueblo del cargador del año. Guatemala: Fundación Yax Te', Plumsock Mesoamerican Studies, Centro de Investigaciones Regionales de Mesoamérica.

Landa, fray Diego

2003 Relación de las cosas de Yucatán. México: Consejo Nacional para la Cultura y las Artes.

Lincoln, Jakson Steward

1942 "The Maya Calendar of the Ixil of Guatemala", Contributions to American Anthropology and History, 38: 97-128.

Macleod, Morna

2013 "Mayan Calendrics in Movement in Guatemala: Mayan Spiritual Guides or Day-Keepers Understandings of 2012", The Journal of Latin American and Caribbean Anthropology, 18 (3): 447-464. Dol: https://doi.org/10.1111/ jlca.12041. 
Martínez González, Roberto

2010 "Nahuales, nahualismo y nahualólogos", Iniciaciones, trances, sueños... Investigaciones sobre el chamanismo en México, pp. 413-440, Antonella Fagetti (coord.). México: Benemérita Universidad Autónoma de Puebla, Plaza y Valdez.

Martínez Hernández, Juan

1926 Paralelismo entre los calendarios maya y azteca. Su correlación con el calendario juliano. México: Compañía Tipográfica Yucateca.

Médicos Descalzos Chinique

2012 ¿Yab'il xane K'oqil? ¿Enfermedades o consecuencias? Seis psicopatologías identificadas y tratadas por los terapeutas Maya'ib' K'iche'ib. Guatemala: Asociación Médicos Descalzos Chinique, Cholsamaj.

Mendizábal, Sergio

2007 El encantamiento de la realidad. Conocimientos mayas en prácticas sociales de la vida cotidiana. Guatemala: Programa de Educación Intercultural Multilingüe de Centroamérica, Universidad Rafael Landívar.

Molesky Poz, Jean

2006 Contemporary Maya Spirituality. Austin: University of Texas Press.

Morales Choy, Luis.

2016 Na’oj Maya Aq’om. Sabiduría Médica Maya. Guatemala: Cholsamaj.

Pédron Colombani, Sylvie

2008 "Diversificación y competencia religiosa en Guatemala: entre pentecostalismo y cultos 'neotradicionales'”, Sociedade e Estado, 23 (2): 355-379. Dol: https://doi.org/10.1590/S0102-69922008000200006.

Pérez Suárez, Tomás

2012 "La escritura istmeña o epiolmeca como antecedente de la maya: una revisión histórica”, Revista Digital Universitaria, 13 (11): 1-16.

Piazza, Rosalba

2012 El cuerpo colonial. Medicina y tradiciones del cuidado entre los maya-k'iche' de Totonicapán, Guatemala. Guatemala: Asociación para el Avance de las Ciencias Sociales en Guatemala.

Piedrasanta, Ruth

2009 Los chuj: unidad y rupturas en su espacio. Guatemala: Armar.

Rodríguez Cano, Laura

2006 "Los registros del tiempo en el México prehispánico", Gran historia gráfica de México ilustrada. Tomo I: El mundo prehispánico, pp. 301-320, Lorenzo Ochoa (coord.). México: Planeta, Consejo Nacional para la Cultura y las Artes, Instituto Nacional de Antropología e Historia. 
Romero Florián, Sergio

2000 Estudio comparativo de dos calendarios coloniales mayas del siglo XVII, tesis de licenciatura en Antropología. Guatemala: Universidad del Valle de Guatemala.

Rupflin Alvarado, Walburga

1999 El Tzolkin es más que un calendario. Guatemala: Fundación Centro de Investigación y Documentación Maya.

Sac Coyoy, Audelino

2007 El calendario sagrado maya, método para el cómputo del tiempo. Quetzaltenango: Universidad Rafael Landívar.

Tedlock, Barbara

1982 Time and the Highland Maya. Albuquerque: University of New Mexico Press.

Thompson, Eric J.

1927 "A Correlation of the Maya and European Calendars", Field Museum of Natural History, Anthropological Series, 17 (1): 5-22. Dol: https://doi.org/10.5962/bhl. title.2720.

1975 Historia y religión de los mayas. México: Siglo Veintiuno.

Urdapilleta, Jorge y Felipe Kajkan Mejía Sepet

2015 "El bastón rojo se sostiene: conocimiento cultural del pueblo Kaqchikel”, Pueblos y Fronteras, 10 (19): 109-141. Dol: https://doi.org/10.22201/ cimsur.18704115e.2015.19.47.

Van Akkeren, Ruud

2012 Xib'alb'a y el nacimiento del Nuevo Sol, una visión posclásica del colapso maya. Guatemala: Piedrasanta.

Villa Rojas, Alberto

1967 "Los lacandones: su origen, costumbres y problemas vitales", América Indígena, 27 (1): 25-53.

2018 "Los conceptos de espacio y tiempo entre los grupos mayances contemporáneos", Tiempo y realidad en el pensamiento maya, pp. 121-163, Miguel León Portilla (ed.). $4^{\mathrm{a}}$ edición. México: Universidad Nacional Autónoma de México.

Weeks, John M., Frauke Sachse y Christian M. Prager

2009 Maya Daykeeping: Three Calendars from Highland Guatemala. Boulder: University Press of Colorado.

Zapil Xivir, Juan

2007 Aproximación lingüística y cultural a los 20 nawales del calendario maya practicado en Momostenango, Totonicapán, tesis de licenciatura en Linguíística. Guatemala: Universidad Rafael Landívar. 
Eréndira Juanita Cano Contreras. Mexicana. Licenciada en Biología por la Universidad Autónoma de Aguascalientes, maestra en Recursos Naturales y Desarrollo Rural por El Colegio de la Frontera Sur, doctora en Ecología y Desarrollo Sustentable en El Colegio de la Frontera Sur, sede San Cristóbal de Las Casas. Sus temas de interés son etnobiología, bioculturalidad, cosmovisión mesoamericana, panmayismo, etnomedicina y sistemas productivos tradicionales, entre otros, y desarrolla investigación sobre mujeres y medicina maya en las tierras altas de Guatemala. Entre sus publicaciones recientes se encuentran "Caminando los solares latinoamericanos: memorias interculturales y resistencia epistémica en territorios de esperanza", "La construcción de la noción de Cosmovisión Maya en Guatemala" y "Latin American Society of Ethnobiology's Code of Ethics", las últimas dos en coautoría.

erecano@gmail.com ercano@ecosur.edu.mx

Erin I. J. Estrada Lugo. Mexicana. Licenciada en Biología por la Universidad Nacional Autónoma de México, maestra en Etnobotánica por el Colegio de Postgraduados y doctora en Antropología Social por la Universidad Iberoamericana. Adscrita al Departamento de Agricultura, Sociedad y Ambiente de El Colegio de la Frontera Sur, sede San Cristóbal de Las Casas. Sus principales temas de interés son la organización social y la apropiación del territorio en el uso de los recursos naturales en las sociedades campesinas indígenas, familia, grupo doméstico y parentesco, normas locales y derecho consuetudinario maya, entre otras. Participa en el proyecto "Community Conservation Research Network: Exploring LocalLevel Enviromental Stewardship across Land and Sea". Entre sus publicaciones recientes se encuentran "Socialización de conocimientos del café orgánico en el contexto de espacios colectivos de grupos localizados de parentesco tseltales, Tenejapa, Chiapas", "El mimbre: una oportunidad de diversificación y desarrollo en las comunidades mam del Soconusco, Chiapas, México" y "La construcción de lo público-colectivo desde las unidades domésticas en el municipio de Bacalar, Quintana Roo", en coautoría.

eestrada@ecosur.mx

Jaime Tomás Page Pliego. Mexicano. Médico cirujano por la Universidad Nacional Autónoma de México, maestro en Medicina Social por la Universidad Autónoma Metropolitana y doctor en Antropología Social por la Universidad Nacional Autónoma de México. Adscrito al Centro de Investigaciones Multidisciplinarias sobre Chiapas y la Frontera Sur de la misma universidad. Sus principales temas de interés son la cosmovisión, etnomedicina, enfermedades del rezago y emergentes en 
población originaria y sistemas y estrategias en salud. Desarrolla los proyectos "Experiencias en la ejecución de un Sistema Local de Salud (silos) inscrito en la epidemiología sociocultural, en Candelaria el Alto, Carranza, Chiapas" y "Representaciones sobre la visión del mundo en Zitim, Huixtán y Nuevo Tepeyac, Villa de Las Rosas, Chiapas". Entre sus publicaciones recientes se encuentran "Subjetividades sobre la causalidad de diabetes mellitus entre seis tseltales de la cabecera municipal de Tenejapa, Chiapas", "La construcción de la noción de cosmovisión maya en Guatemala" y Vivir sufriendo de azúcar: representaciones sociales sobre la diabetes mellitus en tres localidades de los Altos de Chiapas, las dos últimas en coautoría.

jaimepagepliego@comunidad.unam.mx

Egleé L. Zent. Venezolana. Licenciada en Historia del Arte por la Universidad de los Andes, maestra en Biología por el Instituto Venezolano de Investigaciones Científicas, maestra en Artes por la Universidad de California-Berkley y doctora en Antropología por la Universidad de Georgia, efuu. Adscrita al Instituto Venezolano de Investigaciones Científicas, Centro de Antropología, Laboratorio de Ecología Humana. Sus principales temas de interés son la ecología, conservación, biodiversidad, metodologías participativas, estructura y dinámica de poblaciones, etnobiología, etnobotánica y ecología humana. Desarrolla los proyectos "Fitoecología y ecogonía del páramo, Mérida, Venezuela" y "Nï jotï aiye: jkyo jkwainï. libro comunitario de los jodii, su historia y ethos". Entre sus últimas publicaciones se encuentran "Species Distribution Modelling: Contrasting Presence-Only Models with Plot Abundance Data", "Persistent Effects of Pre-Columbian Plant Domestication on Amazonian Forest Composition" y "Ebojto: plantas trepadoras entre los Jotï, Guayana Venezolana”, todas en coautoría.

elzent.7@gmail.com 